\title{
RESISTÊNCIA MECÂNICA E À UMIDADE DE PAINÉIS AGLOMERADOS COM PARTÍCULAS DE MADEIRA DE DIFERENTES DIMENSÕES
}

\author{
MECHANICAL STRENGTH AND DIMENSIONAL STABILITY OF PARTICLEBOARD MADE WITH \\ DIFFERENT PARTICLE SIZES
}

\author{
Clovis Roberto Haselein ${ }^{1}$ Leandro Calegari ${ }^{2}$ Marcos Vinicius Barros ${ }^{3}$ Cristiano Hack ${ }^{3}$ \\ Éverton Hillig ${ }^{4}$ Dalva T. Pauleski ${ }^{3}$ Fernanda Pozzera $^{5}$ \\ RESUMO
}

Neste trabalho, foram confeccionadas e testadas chapas aglomeradas estruturais utilizando part ículas de Pinus elliottii Engelm com dimensões nominais de 110, 75 e $40 \mathrm{~mm}$ de comprimento, 0,5 e 1,0 mm de espessura e $20 \mathrm{~mm}$ de largura. As partículas foram orientadas ao acaso em moldes sem-fundo com dimensões de $50 \times 50 \times 20 \mathrm{~cm}$. Os colchões foram prensados a $180^{\circ} \mathrm{C}$ por 10 minutos até atingir espessura de $9,5 \mathrm{~mm}$ e densidade de, aproximadamente, $0,7 \mathrm{~g} / \mathrm{cm}^{3}$. O adesivo utilizado foi $8 \%$ (em relação ao peso seco das partículas) de tanino-formaldeído. Foram analisadas as propriedades de flexão estática, ligação interna, resistência ao arrancamento de parafusos, dureza Janka, inchamento em espessura e absorção d'água para 2 e 24 horas de imersão. Todos os testes foram realizados segundo a norma americana ASTM D 1037 (1995). As propriedades de flexão estática (MOR e MOE) aumentaram com o aumento do comprimento e diminuição da espessura das partículas. Já o inchamento em espessura e a resistência ao arrancamento de parafusos aumentaram com o aumento da espessura das part ículas.

Palavras-chave: chapa aglomerada, tanino-formaldeído, geometria das partículas.

\section{ABSTRACT}

In this work, flakeboards made of flakes of pine wood (Pinus elliottii Engelm) with dimensions of 110,75 and $40 \mathrm{~mm}$ long, 0,5 and 1,0 $\mathrm{mm}$ thick and $20 \mathrm{~mm}$ wide were manufactured. The particles were manually and randomly oriented into $50 \times 50 \mathrm{~cm}$ mattress and pressed at $180^{\circ} \mathrm{C}$ for 10 minutes to a board thickness of $9,5 \mathrm{~mm}$ and density of $0,7 \mathrm{~g} / \mathrm{cm}^{3}$. The adhesive used was $8 \%$ tannin-formaldehyde. The boards were tested in static bending, internal bond (IB), screw withdrawal, hardness, water absorption and thickness swelling. All tests were performed according to ASTM D1037 (1995). The bending properties (MOR and MOE) increased with increasing flake length and decreased with increasing flake thickness. Thickness swelling and screw withdrawal increased with flake thickness.

Key words: flakeboard, tannin-formaldehyde, particle geometry.

\section{INTRODUÇÃO}

A geometria das partículas é um dos fatores básicos determinantes das características das chapas de partículas. Em conjunto com a espécie de madeira, o tipo e a quantidade de resina, os aditivos, a estrutura da placa formada pela orientação das partículas, a disposição das camadas e as condições de prensagem do colchão determinam as propriedades das chapas aglomeradas.

Conforme Maloney (1989), as propriedades mecânicas como resistência e rigidez à flexão, resistência à tração paralela e perpendicular à superfície (ligação interna), resistência ao arrancamento de

1. Engenheiro Florestal, PhD., Professor do Departamento de Ciências Florestais, Centro de Ciências Rurais, Universidade Federal de Santa Maria, CEP 97105-900, Santa Maria (RS). haseleic@ ccr.ufsm.br

2. Acadêmico do Curso de Graduação em Engenharia Florestal, Centro de Ciências Rurais, Universidade Federal de Santa Maria, CEP 97105-900, Santa Maria (RS). Bolsista de Iniciação Científica da FAPERGS.

3. Acadêmico do Curso de Graduação em Engenharia Florestal, Centro de Ciências Rurais, Universidade Federal de Santa Maria, CEP 97105-900, Santa Maria (RS).

4. Engenheiro Florestal, M.Sc., Professor do Departamento de Ciências Exatas e da Natureza e de Engenharia Química, Universidade de Caxias do Sul, CEP 95034-190, Caxias do Sul (RS).

5. Engenheira Florestal, Mestranda pelo Programa de Pós-Graduação em Engenharia Florestal, Centro de Ciências Rurais, Universidade Federal de Santa Maria, CEP 97105-900, Santa Maria (RS). 
parafusos e pregos são afetadas pela geometria das partículas. A geometria das partículas também influi nas propriedades físicas das chapas, como absorção de umidade (na forma líquida ou de vapor), mudanças correspondentes em dimensões e características da superfície.

Segundo Kollman et al. (1975), a dimensão das partículas é uma variável de merecida importância sendo seu tipo e tamanho os principais fatores a serem considerados. Brito e Peixoto (2000), avaliando duas granulométrias de partículas de Pinus taeda, afirmam que, uma vez alterada a geometria ou granulométria das partículas, há a necessidade também de se averiguar outras variáveis do processo, sobretudo a melhor proporção de adesivo a ser empregada.

O termo "partícula" é definido no padrão ASTM como o componente agregado de uma chapa de partículas de madeira ou outro material de lignocelulósico, incluindo todas as menores subdivisões da madeira, fabricadas por meios mecânicos. Os tipos de partículas mais comumente usados nos processos de composição de chapas são flocos, maravalhas e fibras, empregadas em tamanhos variados. Part ículas geradas por moinho de martelos são produzidas de resíduos da industrialização da madeira ou de matéria-prima proveniente de florestas plantadas.

Para Vital et al. (1992), o uso de partículas curtas e espessas beneficia a resistência à tração perpendicular, porém ocasiona redução da tensão de ruptura e do módulo de elasticidade à flexão estática. Essas duas propriedades são melhoradas quando se aumenta o comprimento das partículas. Esse efeito é resultado de alterações na parte superficial e na flexibilidade das partículas, do consumo relativo de adesivo e da área de contato entre as partículas.

Moslemi (1974) estabelece que partículas finas, pequenas e flexíveis geram superfícies livres de vazios. A flexibilidade das partículas não-somente tem influência direta no aspecto da superfície e das bordas como também determina, em grande parte, o grau de contato partícula-partícula e, portanto, influencia as propriedades físico-mecânicas das chapas.

Entretanto, as diversas propriedades das chapas são afetadas de forma diferenciada pelas variações nas dimensões das partículas. Assim, Brumbaugh (1960) e Gatchell et al. (1966) observaram que as partículas longas e finas melhoraram a estabilidade dimensional das chapas. Segundo Vital e Wilson (1980), o efeito da espessura das partículas sobre a estabilidade dimensional das chapas depende também de seu comprimento.

Vital et al.(1992), em seus estudos sobre o efeito da geometria da partícula, constatou que não houve tratamento que melhorasse simultaneamente todas as propriedades mecânicas e a estabilidade dimensional das chapas. No entanto, observou que, mantendo constantes as demais vari áveis, o aumento da espessura das partículas resultou sempre na redução dos valores das propriedades relacionadas à flexão estática. $\mathrm{O}$ autor defende que as partículas mais finas distribuem melhor as tensões, além de produzirem menor quantidade de espaços vazios no interior das chapas, produzindo chapas mais resistentes. Estabelece ainda que para se produzir chapas de partículas mais rígidas, resistentes à flexão e com maior estabilidade dimensional, é necessário empregar partículas mais finas. Para maior resistência à tração perpendicular, é necessário o uso de partículas mais espessas.

Brito e Peixoto (2000) descreveram que a razão entre o comprimento e a espessura das partículas (coeficiente de esbeltez) tem uma relação direta com o MOE, e que esse valor deve ser, no mínimo, de 200.

Os objetivos do presente projeto de pesquisa foram testar a resistência mecânica e a resistência à umidade de painéis aglomerados colados com tanino-formaldeído, contendo diferentes geometrias de partículas de madeira.

\section{MATERIAL E MÉTODOS}

O presente estudo foi conduzido no Laboratório de Produtos Florestais. A madeira para a produção das partículas foi obtida de três exemplares de Pinus elliottii Engelm, selecionadas ao acaso, de um povoamento florestal com 15 anos de idade, localizado no Campus da UFSM.

De cada árvore, foram retiradas as duas primeiras toras de, aproximadamente, 2,5 $\mathrm{m}$, at é a altura de $5 \mathrm{~m}$. Discos foram retirados dessas mesmas posições para a determinação da massa específica básica. As 
toras foram posteriormente transformadas em tábuas, as quais possuíam espessura média de $19,10 \mathrm{~mm}$. Essas tábuas foram armazenadas em um tanque com água. Após saturadas, cada tábua foi seccionada no sentido transversal em comprimentos médios de 40, 75 e $110 \mathrm{~mm}$, obtendo-se, desse modo, baguetas de três diferentes comprimentos. Ainda saturadas e a uma temperatura em torno de $50^{\circ} \mathrm{C}$, essas baguetas foram transformadas em partículas com espessuras médias de 0,50 e 1,00 mm, utilizando um gerador de partículas de laboratório.

Desse modo, os tratamentos basearam-se na utilização de partículas com três diferentes comprimentos e duas diferentes espessuras. A largura das part ículas foi mantida constante, determinada pela espessura média das tábuas. Os tratamentos investigados são caracterizados na Tabela 1.

TABELA 1: Dimensões nominais das partículas utilizados na confecção das chapas para os tratamentos.

TABLE 1: Particle dimensions used for the treatments in the board manufacture.

\begin{tabular}{c|c|c|c}
\hline \multirow{2}{*}{ Tratamentos } & \multicolumn{3}{|c}{ Dimensões Nominais das Partículas (mm) } \\
\cline { 2 - 4 } & Comprimento & Largura & Espessura \\
\hline 1 & 110,0 & 20,0 & 0,5 \\
2 & 110,0 & 20,0 & 1,0 \\
3 & 75,0 & 20,0 & 0,5 \\
4 & 75,0 & 20,0 & 1,0 \\
5 & 40,0 & 20,0 & 0,5 \\
6 & 40,0 & 20,0 & 1,0 \\
\hline
\end{tabular}

As partículas foram secadas ao ar e armazenadas em sacos de polietileno. Aproximadamente 12 horas antes de sua utilização, foram colocados em estufa com circulação forçada de ar, à temperatura de $60^{\circ} \mathrm{C}$, condições essas que proporcionaram uma umidade de equilíbrio de, aproximadamente, 3\% (base seca).

As chapas foram produzidas aleatoriamente com três repetições por tratamento, excetuando-se os tratamentos 1 e 3, para os quais foram feitas quatro repetições. Desse modo, foi fabricado um total de 20 chapas, com uma densidade nominal de $0,7 \mathrm{~g} / \mathrm{cm}^{3}$.

$\mathrm{O}$ adesivo utilizado foi à base de tanino-formaldeído, na proporção 100:10 respectivamente (partes por peso de sólidos não-voláteis). O tanino possuía um teor de sólidos de $37 \%$ e teve seu $\mathrm{pH}$ corrigido para 8 com o uso de hidróxido de sódio. O formaldeído utilizado, por sua vez, apresentava $40 \%$ de sólidos.

Os teores de parafina (emulsão com 52\% de sólidos) e adesivo foram mantidos constantes, utilizando-se 1 e $8 \%$ respectivamente, baseando-se no peso seco das partículas. A umidade nominal do colchão foi estabelecida em 18\% (base seca). Os materiais utilizados foram misturados com o aux ílio de um tambor de laboratório dotado de uma pistola acionada por ar comprimido. Após a mistura, para cada chapa confeccionada, retirou-se do tambor rotativo três amostras de partículas para a determinação do teor de umidade.

Os colchões foram formados manualmente e pré-prensados em um molde de madeira de $50 \mathrm{x} 50 \mathrm{x}$ $20 \mathrm{~cm}$, à temperatura ambiente, durante um período de, aproximadamente, um minuto. Chapas de aço galvanizado foram colocadas nas faces superior e inferior do colchão. Para um melhor acabamento e evitar aderência do painel ao aço, ainda se utilizaram folhas de papel vegetal em ambas as faces do colchão, evitando o contato direto da chapa aglomerada com a chapa de aço.

A prensagem definitiva do colchão foi feita em uma prensa hidráulica marca OMECO, com capacidade para 100 toneladas, durante 10 minutos, com press ão específica de $27 \mathrm{kgf} / \mathrm{cm}^{2}$ e temperatura de $180^{\circ} \mathrm{C}$. A espessura final das chapas aglomeradas foi determinada em $9,5 \mathrm{~mm}$ por meio do uso de duas barras de aço dessa espessura colocadas em lados opostos do colch ão, as quais limitavam o fechamento total da prensa.

Logo após retiradas da prensa, as chapas foram levadas à câmara climatizada, onde foram mantidas a $20^{\circ} \mathrm{C}$ e $65 \%$ de umidade relativa, até peso constante. Após esquadrejamento, mediu-se a espessura média de cada chapa. 
Os corpos-de-prova foram confeccionados após as chapas terem sido levemente lixadas. Para a determinação da resistência à tração perpendicular, foram utilizadas quatro amostras por chapa, enquanto que para a determinação da resistência à flexão estática, variação dimensional em espessura e absorção d'água foram utilizadas duas amostras para cada teste.

Para a realização dos testes de flexão estática, resistência ao arrancamento de parafusos, ligação interna e dureza Janka, utilizou-se uma máquina universal de ensaios, hidráulica, marca AMSLER, com capacidade de 20 toneladas. A avaliação da absorção d'água e do inchamento em espessura foi realizada após 2 e 24 horas de imersão.

Todos os testes foram realizados segundo a norma ASTM D 1037 (1995). Os resultados foram interpretados com o auxílio de um pacote estatístico, relacionando as propriedades das chapas aos tratamentos, pelos testes de média e regressão.

\section{RESULTADOS E DISCUSSÃO}

A madeira utilizada apresentou densidade básica igual a $0,48 \mathrm{~g} / \mathrm{cm}^{3}$ e, mesmo saturada e aquecida, mostrou-se difícil de ser transformada de bagueta em partículas. Houve dificuldade em obter-se partículas com as espessuras nominais desejadas, uma vez que a mesma regulagem de facas produzia part ículas com diferentes espessuras, dependendo do comprimento da partícula.

Da mesma forma que Hillig (2000), um dos principais problemas verificado na fabricação das chapas foi o controle do teor de umidade do colchão e, conseqüentemente, do teor de adesivo. Durante a mistura dos componentes formadores do colchão no tambor rotativo, observou-se que, aplicando-se quantidades iguais desses componentes, colchões formados por partículas mais curtos e espessos apresentaram menor teor de umidade quando comparados aos colchões formados por partículas mais longas e finas. Essa diferença se deve aos diferentes volumes proporcionados pelas diferentes partículas. Para partículas de menores comprimentos e maiores espessuras o volume do colchão é menor, reduzindo a área de recebimento dos componentes em emulsão (tanino-formaldeído, parafina e água) e, conseqüentemente, aumentando as perdas, uma vez que considerável percentagem desses componentes ficam aderidos ao fundo do tambor. Brito e Peixoto (2000) também concluíram que existe uma influência do tipo de partícula sobre a proporção de adesivo. A fim de evitar uma variação significativa na quantidade dos componentes em emulsão aplicados com relação a cada tratamento, procurou-se corrigir seus pesos com diferentes margens, por tentativa, e baseando-se no teor de umidade do colchão determinado após a mistura. Em colchões de menores volumes, maiores margens foram necessárias quando comparados a colchões de maiores volumes. Desse modo, conseguiu-se homogeneizar o teor de umidade dos colchões e, provavelmente, o teor de adesivo. As dimensões médias reais das partículas utilizadas e demais características das chapas, em função dos tratamentos, são apresentadas na Tabela 2.

TABELA 2: Valores reais médios das partículas utilizadas e demais características das chapas com relação aos tratamentos.

TABLE 2: Average particle sizes used and board characteristics in relation to the treatments.

\begin{tabular}{|c|c|c|c|c|c|c|}
\hline \multirow[t]{2}{*}{ Tratamentos } & \multicolumn{2}{|c|}{$\begin{array}{c}\text { Dimensão dos Flocos }{ }^{2} \\
(\mathrm{~mm})\end{array}$} & \multirow{2}{*}{$\begin{array}{c}\text { Umidade } \\
\text { do Colchão } \\
(\%)\end{array}$} & \multirow{2}{*}{$\begin{array}{c}\text { Espessura } \\
\text { das Chapas }{ }^{3} \\
(\mathrm{~mm})\end{array}$} & \multirow[t]{2}{*}{$\begin{array}{l}\text { Dens } \\
\left(\mathrm{g} / \mathrm{cm}^{3}\right)\end{array}$} & \multirow{2}{*}{$\begin{array}{c}\text { Umidade } \\
\text { Equilíbrio }^{4} \\
(\%)\end{array}$} \\
\hline & Comprimento & Espessura & & & & \\
\hline 1 & 116,96 & 0,44 & $17,30 \mathrm{a}^{1}$ & $10,23 \mathrm{a}$ & 0,72 & $8,60 \mathrm{a}$ \\
\hline 2 & 116,96 & 0,91 & $16,80 \mathrm{a}$ & $11,33 \mathrm{a}$ & $0,72 \mathrm{bc}$ & $8,38 \mathrm{a}$ \\
\hline 3 & 73,83 & 0,57 & $16,70 \mathrm{a}$ & $09,60 \mathrm{a}$ & $0,69 \mathrm{ab}$ & $8,30 \mathrm{a}$ \\
\hline 4 & 77,32 & 1,08 & $16,57 \mathrm{a}$ & $10,43 \mathrm{a}$ & $0,70 a b c$ & $8,70 \mathrm{a}$ \\
\hline 5 & 40,20 & 0,50 & $16,67 \mathrm{a}$ & $10,20 \mathrm{a}$ & 0,68 & $8,10 \mathrm{a}$ \\
\hline 6 & 36,34 & 1,12 & $16,67 \mathrm{a}$ & $10,63 \mathrm{a}$ & $0,69 a b c$ & $8,60 \mathrm{a}$ \\
\hline
\end{tabular}

Em que: Valores seguidos pela mesma letra não diferem estatisticamente, ao nível de 5\% de probabilidade, pelo teste Least Significant Difference (LSD) de Fisher; ${ }^{2}$ Média de 230 partículas por tratamento; ${ }^{3}$ Determinada após condicionamento, em quatro pontos nas arestas das chapas; Dens = densidade (peso seco/volume ao teor de umidade de equilíbrio); ${ }^{4} 20^{\circ} \mathrm{C}$ e $65 \%$ UR. 
A espessura média das chapas, após equilíbrio a $20^{\circ} \mathrm{C}$ e $65 \%$ de umidade relativa, foi de $10,44 \mathrm{~mm}$. Não houve diferença significativa na espessura por causa dos tratamentos, considerando o teste $\mathrm{F}$, a um nível de confiança de $95 \%$. O mesmo fato ocorreu com relação ao teor de umidade do colchão e à umidade de equilíbrio dos corpos-de-prova utilizados, os quais apresentaram valores médios de 16,8 e 8,45\% respectivamente.

A densidade das chapas, determinada pela razão entre seu peso seco ( $0 \%$ de umidade) e seu volume à umidade de equilíbrio $\left(20^{\circ} \mathrm{C}\right.$ e $\left.65 \% \mathrm{UR}\right)$, foi de, aproximadamente, $0,70 \mathrm{~g} / \mathrm{cm}^{3}$. Essa definição, embora não- -tradicional, diminuiu a variabilidade dos dados, descontando pequenas oscilações na umidade de equilíbrio das chapas. A densidade aparente pode ser facilmente obtida corrigindo os valores da Tabela 2 pela respectiva umidade de equilíbrio.

Procurou-se manter constante a densidade durante a fabricação das chapas. No entanto, isto nem sempre foi possível, conforme indicado pela Análise da Variância, a qual demonstrou diferença estatística significativa. Por essa razão, a densidade foi corrigida pela análise de covariância.

Já os valores médios das propriedades físicas e mecânicas das chapas, em função dos tratamentos, são apresentados nas Tabelas 3 e 4, respectivamente.

TABELA 3: Contraste relativo às propriedades mecânicas das chapas para os tratamentos (corrigido para o efeito da densidade das chapas).

TABLE 3: Contrast related to the board mechanical properties for the treatments (correct to the effect of board density).

\begin{tabular}{c|c|c|c|c|c}
\hline Tratamentos & $\begin{array}{c}\text { MOE } \\
\left(\mathrm{kgf} / \mathrm{cm}^{2}\right)\end{array}$ & $\begin{array}{c}\text { MOR } \\
\left(\mathrm{kgf} / \mathrm{cm}^{2}\right)\end{array}$ & $\begin{array}{c}\text { Ligação Interna } \\
\left(\mathrm{kgf} / \mathrm{cm}^{2}\right)\end{array}$ & $\begin{array}{c}\text { Arrancamento de } \\
\text { parafusos }(\mathrm{kgf})\end{array}$ & $\begin{array}{c}\text { Dureza Janka } \\
(\mathrm{kgf})\end{array}$ \\
\hline 1 & $28256 \mathrm{bc}$ & $232,08 \mathrm{c}$ & $2,18 \mathrm{a}$ & $148,96 \mathrm{bc}$ & $586,59 \mathrm{a}$ \\
2 & $30303 \mathrm{c}$ & $232,34 \mathrm{c}$ & $1,89 \mathrm{a}$ & $126,45 \mathrm{abc}$ & $622,16 \mathrm{a}$ \\
3 & $25977 \mathrm{bc}$ & $173,34 \mathrm{~b}$ & $2,04 \mathrm{a}$ & $127,48 \mathrm{ab}$ & $641,12 \mathrm{a}$ \\
4 & $24668 \mathrm{~b}$ & $173,26 \mathrm{~b}$ & $1,93 \mathrm{a}$ & $155,67 \mathrm{c}$ & $623,53 \mathrm{a}$ \\
5 & $29778 \mathrm{c}$ & $196,26 \mathrm{bc}$ & $2,86 \mathrm{~b}$ & $110,28 \mathrm{a}$ & $546,61 \mathrm{a}$ \\
6 & $19006 \mathrm{a}$ & $106,02 \mathrm{a}$ & $1,85 \mathrm{a}$ & $155,35 \mathrm{bc}$ & $501,67 \mathrm{a}$ \\
\hline
\end{tabular}

Em que: Valores seguidos pela mesma letra não diferem estatisticamente, ao nível de 5\% de probabilidade, pelo teste Least Significant Difference (LSD) de Fisher.

TABELA 4: Contraste relativo às propriedades físicas das chapas em função dos tratamentos (corrigido para o efeito da densidade das chapas).

TABLE 5: Contrast related to the board physical properties for the treatments (correct to the effect of board density).

\begin{tabular}{c|c|c|c|c}
\hline \multirow{2}{*}{ Tratamentos } & \multicolumn{2}{|c|}{ Absorção d'água (\%) } & \multicolumn{2}{c}{ Inchamento em Espessura (\%) } \\
\cline { 2 - 5 } & $2 \mathrm{H}$ & $24 \mathrm{H}$ & $2 \mathrm{H}$ & $24 \mathrm{H}$ \\
\hline 1 & $12,20 \mathrm{bc}^{1}$ & $56,74 \mathrm{a}$ & $4,48 \mathrm{a}$ & $27,43 \mathrm{a}$ \\
2 & $8,81 \mathrm{ab}$ & $60,46 \mathrm{a}$ & $4,11 \mathrm{a}$ & $32,72 \mathrm{abc}$ \\
3 & $8,83 \mathrm{ab}$ & $66,45 \mathrm{a}$ & $3,22 \mathrm{a}$ & $29,18 \mathrm{ab}$ \\
4 & $7,98 \mathrm{a}$ & $57,87 \mathrm{a}$ & $3,47 \mathrm{a}$ & $33,90 \mathrm{bc}$ \\
5 & $14,30 \mathrm{c}$ & $64,54 \mathrm{a}$ & $3,47 \mathrm{a}$ & $28,02 \mathrm{a}$ \\
6 & $11,87 \mathrm{abc}$ & $60,70 \mathrm{a}$ & $4,72 \mathrm{a}$ & $35,43 \mathrm{c}$ \\
\hline
\end{tabular}

Em que: Valores seguidos pela mesma letra não diferem estatisticamente, ao nível de 5\% de probabilidade, pelo teste Least Significant Difference (LSD) de Fisher.

Não houve tratamento que melhorasse simultaneamente todas as propriedades mecânicas e físicas. De um modo geral, mantendo-se constante as demais variáveis, o aumento na espessura das partículas provocou uma redução dos valores das propriedades relacionadas à flexão estática (MOR e MOE). Esse 
resultado coincide com as citações de Brito e Peixoto (2000), os quais descreveram que as propriedades de flexão estática das chapas são inversamente proporcionais à espessura das partículas.

A resistência à tração perpendicular (ligação interna) foi menor nas chapas produzidas com partículas mais espessas, apesar dessa diferença não ser estatisticamente significativa em alguns casos. Observou-se também que essa variável apresenta uma certa tendência de diminuição com a redução dos comprimentos das partículas.

Vital et al. (1992), no entanto, avaliando o efeito da geometria das partículas sobre as propriedades físico-mecânicas de chapas aglomeradas, concluiram que maior resistência à tração é obtida com o aumento da espessura e diminuição do comprimento dos flocos. Brito e Peixoto (2000) também concluíram que chapas feitas com partículas menores apresentaram uma maior resistência à tração perpendicular quando comparadas à partículas maiores. Descrevem que partículas menores são responsáveis por uma melhor uniformização do material e pela formação de menores espaços internos.

Do mesmo modo que concluído por Vital et al. (1992), a resistência ao arrancamento de parafusos foi influenciada significantemente pelos tratamentos, porém, sem qualquer tendência lógica.

A dureza Janka não foi afetada pela geometria das partículas, uma vez que a análise de variância não demonstrou diferença estatística significativa entre os tratamentos $(\mathrm{P}=79 \%)$.

A absorção d'água após 24 horas de imersão não foi influenciada pelo comprimento das partículas. Para partículas de mesmo comprimento, o aumento na espessura das partículas resultou num maior inchamento em espessura da chapa aglomerada após um período de 24 horas de imersão. Esse resultado coincide com o encontrado por Vital et al. (1992), o qual argumenta que partículas mais finas produzem menor quantidade de espaços vazios no interior das chapas e, conseqüentemente, produziam chapas mais resistentes.

Brito e Peixoto (2000), no entanto, em seu estudo avaliando a granulometria das partículas, citam que melhores valores de inchamento são obtidos nas chapas produzidas com partícula de granulometria maior. Defende que partículas com granulometria maior tem uma menor superfície específica e, conseqüentemente, menor área para absorção d'água.

Como a Análise de Variância nem sempre é uma ferramenta robusta, quando possível foi feita também análise de regressão, relacionando as propriedades das chapas ao comprimento e à espessura das partículas. Modelos significativos foram encontrados para MOR, MOE, arrancamento de parafusos, liga ção interna e inchamento após 24 horas de imersão em água, conforme mostra a Tabela 5 e Figuras 1 e 2.

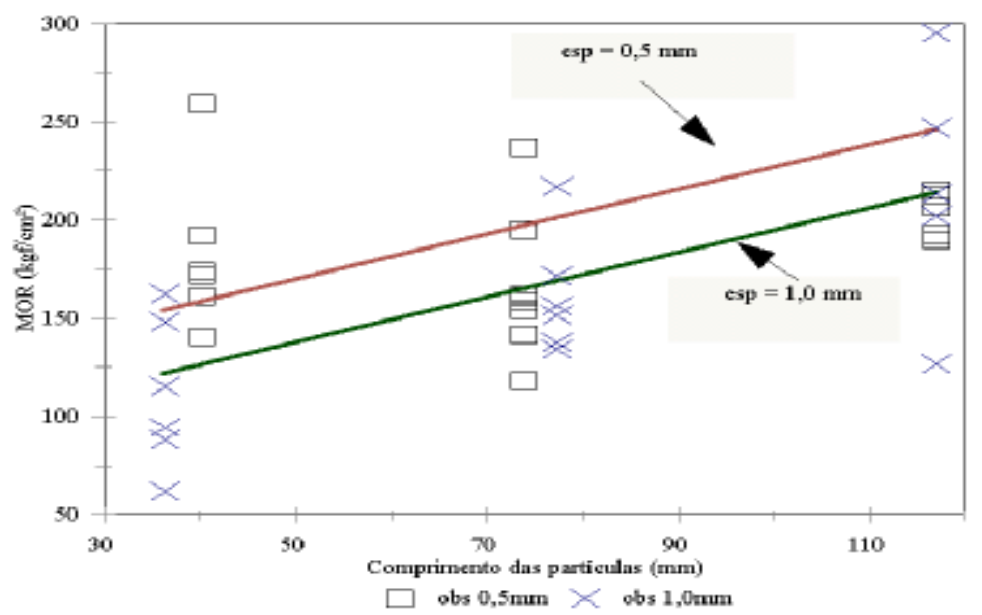

FIGURA 1: MOR observado e estimado em função do comprimento das partículas, para as duas espessuras estudadas.

FIGURE 1: MOR observed and estimated as a function of particle length, for the two particle thicknesses studied. 
TABELA 5: Equações ajustadas para as propriedades das chapas pelo método "stepwise".

TABLE 5: Fitted equations for the board properties by the stepwise method.

\begin{tabular}{|c|c|c|c|c|}
\hline Propriedade & Equação Ajustada & $\mathrm{F}$ & Syx & $\mathrm{R}^{2}$ ajustado $(\%)$ \\
\hline MOR & $\begin{array}{l}\text { - } 354,172+0,956 \text { comp }+740,48 \text { dens } \\
\text { - } 61,37 \text { esp }\end{array}$ & 19,61 & 43,47 & 58,88 \\
\hline MOE & $-30911,4+52,3$ comp $+84397,2$ dens $-6899,2$ esp & 12,69 & 4857 & 47,34 \\
\hline LI & $2,61667-0,680368 \mathrm{esp}$ & 5,09 & 0,6677 & 7,16 \\
\hline $\mathrm{I}_{24}$ & $-22,028+63,015$ dens $+10,92$ esp & 12,63 & 4,51 & 38,60 \\
\hline AP & $-141,61+403,77$ dens & 12,08 & 28,99 & 22,20 \\
\hline
\end{tabular}

Em que: $\mathrm{MOR}=$ módulo de ruptura; $\mathrm{MOE}=$ módulo de elasticidade; comp = comprimento das partículas; esp = espessura das partículas; dens = densidade das chapas; $\mathrm{LI}=$ ligação interna; $\mathrm{I}_{24}=$ inchamento após 24 horas; $\mathrm{AP}=$ arrancamento de parafusos.

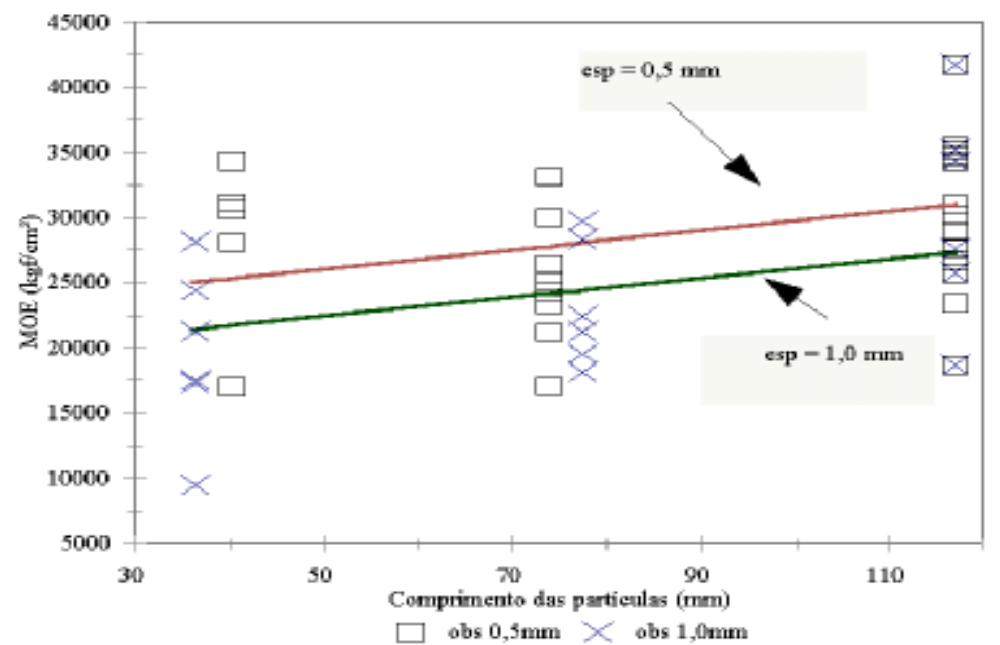

FIGURA 2: MOE observado e estimado em função do comprimento das partículas, para as duas espessuras estudadas.

FIGURE 2: MOE observed and estimated as a function of particle length, for the two thicknesses studied.

A inclusão da densidade melhorou sensivelmente o coeficiente de determinação ajustado para as propriedades, exceto ligação interna, influenciada somente pela espessura das partículas. O coeficiente de determinação para essa propriedade foi muito baixo $(7,16 \%)$, indicando pequena influência dos parâmetros estudados. Para os tipos de partícula estudados, as alternativas seriam aumentar o teor de adesivo ou a massa específica das chapas para que os valores sejam compatíveis com aqueles exigidos comercialmente de acordo com a ANSI (1979).

\section{CONCLUSÕES}

As propriedades de flexão estática (MOR e MOE) das chapas foram diretamente proporcionais ao comprimento e inversamente proporcionais à espessura das partículas.

Já a resistência à tração perpendicular (ligação interna) foi inversamente proporcional ao aumento da espessura das partículas. Os valores encontrados para essa propriedade foram bem abaixos daqueles requeridos para comercialização.

A dureza Janka, a absorção d'água, após 24 horas, e o inchamento em espessura, após 2 horas de imersão das chapas em água, não foram influenciados pela geometria das partículas.

O inchamento em espessura, após 24 horas de imersão, e a resistência ao arrancamento de parafusos não foram influenciados pelo comprimento das partículas. Entretanto, foram diretamente proporcionais ao aumento da espessura das partículas. 


\section{REFERENCIAS BIBLIOGRÁFICAS}

AMERICAN NATIONAL STANDARDS INSTITUTE. Mat-formed wood particleboard. ANSI A 208.1. New York, 1979.

AMERICAN SOCIETY FOR TESTING AND MATERIALS. Standard Test Methods for Evaluating Properties of Wood-Base Fiber and Particle Panel Materials. ASTM D1037 - 93. Philadelphia, PA, 1995.

BRITO,E.O.; PEIXOTO, G.L. Avaliação da granulometria de partículas de Pinus taeda combinadas com adesivos comerciais para a fabricação de aglomerados. Revista Floresta e Ambiente. Rio de Janeiro, v. 7, n. 1, p. 60-67, jan./dez. 2000.

BRUMBAUGH, J. Effect of flake dimensions on properties of particleboard. Forest Products Journal, v. 10, n. 5, p. 243-246, 1960.

GATCHELL, C.J.; HEEBINK, B.G.; HEFTY, F.V. Influence of components variables on properties of particleboard for exterior use. Forest Products Journal, v. 16, n. 4, p. 46-59, 1966.

HILLIG, E. Qualidade de chapas aglomeradas estruturais, fabricadas com madeiras de Pinus, Eucalipto e Acácia negra, puras ou misturadas, coladas com tanino-formaldeído. 2000. 96p. Dissertação (Mestrado em Engenharia Florestal) - Universidade Federal de Santa Maria, Santa Maria.

KOLLMANN, F.P.T.; KUENZI, E.W.; STAMM, A.J.; Principles of wood science and tecnology II: wood based materials. 2. ed. Berlin; Springer-Verlag, 1975. v. 2.

MALONEY, T. M. Modern particleboard and dry-process fiberboard manufacturing. San Francisco, Califórnia: Miller Freeman Publications, 1989.

MOSLEMI, A. A. Particleboard. Southern: Illinois University Press, 1974. v. 1.

VITAL, B.R.; HASELEIN, C.R.; DELLA LUCIA, R.M. Efeito da geometria das partículas nas propriedades das chapas de madeira aglomerada de Eucalyptus grandis (Hill ex-Maiden). Revista Árvore, Viçosa, v. 16, n. 1, p. 88-96,1992.

VITAL, B.R.; WILSON, J.B. Efeito da forma geométrica dos flocos e partículas, da densidade das chapas e do tipo de adesivo nas propriedades mecânicas das chapas de madeira aglomerada. Revista Árvore, Viçosa, v. 4, n. 2, p. 179-187, 1980 . 